\title{
Mini-Review
}

Theme: Rising Stars in Drug Delivery and Novel Carriers

Guest Editors: Aliasger Salem, Juliane Nguyen and Kristy Ainslie

\section{The Nano-War Against Complement Proteins}

\author{
Zhicheng Wang ${ }^{1,2}$ and Jacob S. Brenner ${ }^{1,2,3}$
}

Received 4 April 2021; accepted 27 July 2021; published online 10 September 2021

Abstract. Targeted drug delivery and nanomedicine hold the potential promise of
delivering drugs solely to target organs or cell types, thus decreasing off-target side effects
and improving efficacy. However, nano-scale drug carriers face several barriers to this goal,
with one of the most formidable being the complement cascade. Complement proteins,
especially C3, opsonize not just the microbes they evolved to contain, but also nanocarriers.
This results in multiple problems, including marking the nanocarriers for clearance by
leukocytes, likely fouling of the targeting moieties on nanocarriers, and release of toxins
which produce deleterious local and systemic effects. Here, we review how complement
achieves its blockade of nanomedicine, which nanocarrier materials properties best avoid
complement, and current and future strategies to control complement to unleash
nanomedicine's potential.

KEYWORDS: complement; nanomedicine; nanoparticle; targeted drug delivery; C3.

\section{INTRODUCTION TO THE COMBATANTS: NANOMEDICINE}

The vast majority of promising drug candidates ultimately fail. These failures occur at multiple stages of development and for multiple reasons, but perhaps the largest cause is off-target side effects. Even poor efficacy is often caused by the necessity of a reduced dose due to dose-limiting side effects.

To combat this pervasive impediment to medicine, the field of targeted drug delivery aims to deliver cargo drugs solely to the target organ or cell type. Such targeted delivery should reduce the total drug mass necessary, and thus reduce off-target side effects. While this idea is often pitched as a new approach, it was actually first realized as a successful drug $>100$ years ago. Paul Ehrlich introduced the idea of targeted drug delivery with his analogy of the "magic bullet" that could find its way through a crowd to hit only an escaping criminal. He then actualized that idea in creating Salversan in the early 1900s (Fig. 1), the first true antibiotic, which virtually cured the widespread scourge of syphilis [1].

\footnotetext{
Responsible Editor: Aliasger Salem, Juliane Nguyen and Kristy Ainslie

${ }^{1}$ Pulmonary, Allergy \& Critical Care Division, Department of Medicine, University of Pennsylvania, 3450 Hamilton Walk, Stemmler Building, Office \#220, Philadelphia, Pennsylvania 19104, USA.

${ }^{2}$ Systems Pharmacology \& Translational Therapeutics Department, University of Pennsylvania, Philadelphia, Pennsylvania 19104, USA.

${ }^{3}$ To whom correspondence should be addressed. (e-mail: Jacob.brenner@pennmedicine.upenn.edu)
}

Salversan was composed of two components that he covalently linked: a targeting moiety (in this case, a small molecule "dye" literally borrowed from the dye industry) that was found in a screen to bind syphilis bacteria and a cargo drug (in this case a general microbicide, arsenic). Salversan dramatically reduced the side effects of arsenic and was wildly popular. Unfortunately, Salversan's success was never replicated for small molecule drugs, likely because the similar size of the targeting moiety and cargo drug caused them to interfere with each other.

To overcome the issues of the targeting moiety and cargo drug sterically hindering each other, nanomedicine was introduced in the 1960s-70s [2, 3]. Nanomedicine adds to the two components of Ehrlich's magic bullet (targeting moiety and cargo drug) a third component: nano-scale drug carriers (nanocarriers). Nanocarriers are typically spheres ranging from $\sim 10$ nanometers $(\mathrm{nm})$ to $\sim 300 \mathrm{~nm}$, filled with cargo drug and possessing targeting moieties on their surface. The earliest and perhaps most commonly studied such targeted nanocarriers are liposomes ( 100 nm lipid bilayers with an aqueous interior) filled with small molecule drugs [3]. Additionally, innumerable variations have been made around this core idea of targeted nanomedicine, including such key ones as: not including targeting moieties, with the nanocarrier providing benefits of solubilization, increased plasma half-life, and "passive" targeting via alterations in the target tissue (all of these being exemplified by the first approved cancer nanomedicine, Doxil) [4]; changing from small molecule to nucleic acid cargo (such as the first siRNA nanocarrier approved, patisiran) [5]; and, of course, using diverse materials for the nanocarrier. By the time of this writing, 


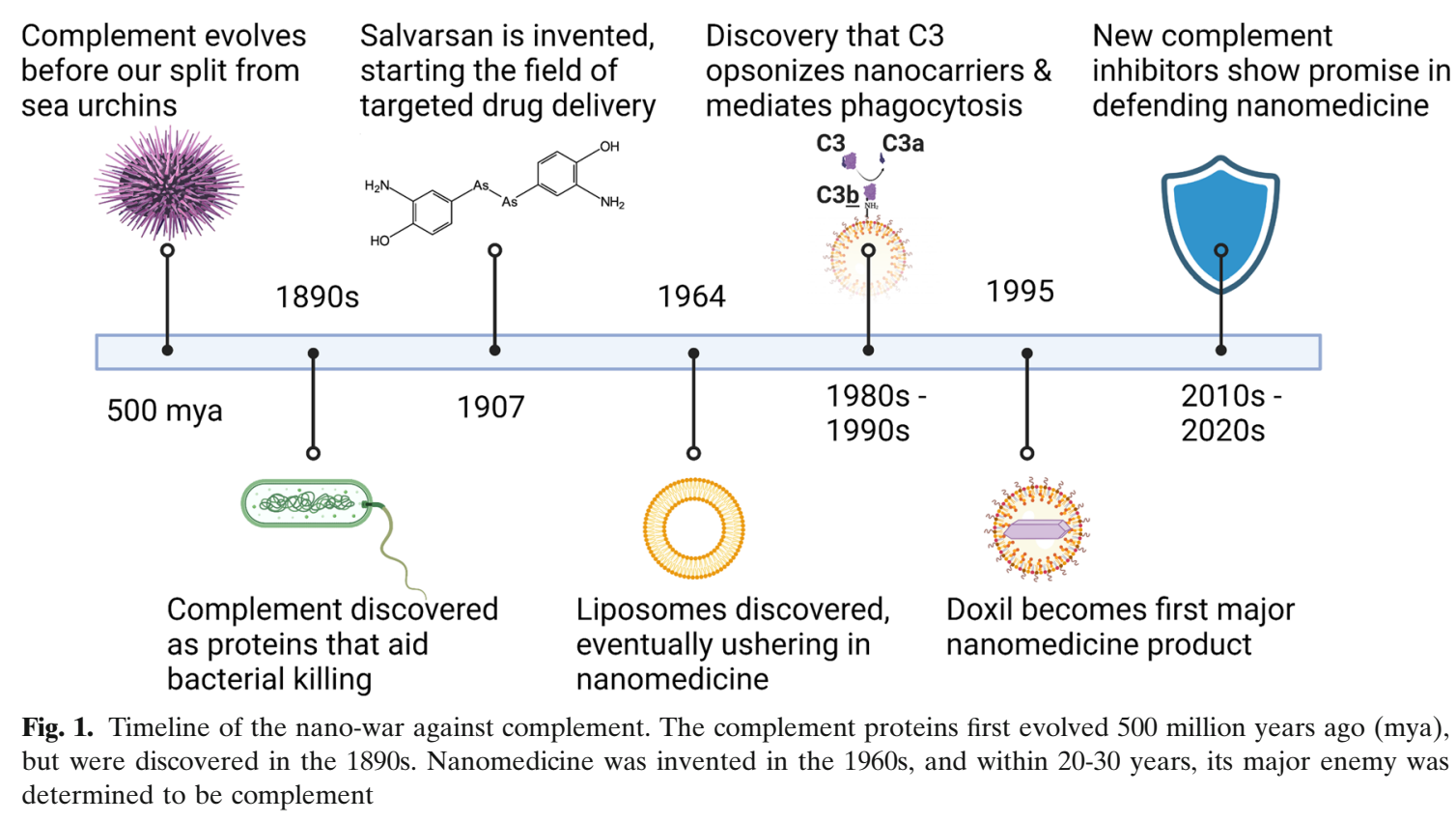

there are now more than 15 FDA-approved medicines composed of nanocarriers. Thus, the introduction of nanocarriers clearly was a successful innovation that is continuing to provide new therapeutics.

However, the introduction of nanocarriers also created a new set of problems. The first-identified problem, and still the biggest, is that the majority of nanoparticles are taken up by the reticuloendothelial system (RES)/monocyte-phagocytic system (MPS) [6]. The RES is classically described as the organ-resident leukocytes (especially Kupffer cells in the liver) that surveil the blood and remove microbes and particulate matter.

Decades later, it was recognized that the RES problem is in large part a consequence of the opsonization of nanocarriers, meaning the nanocarriers are coated (opsonized) by blood proteins. First, it was found that among the opsonins that bound nanocarriers, a set of proteins called "complement" were over-represented [7]. Then, in the 1990s, the complement opsonization of nanocarriers, especially with complement protein $\mathrm{C} 3$, was shown to drive nanocarrier phagocytosis by RES leukocytes [8-13]. Thus, the war between nanocarrier engineers and complement has been a "30-years war," and thus far, we engineers are not winning.

\section{INTRODUCTION TO THE COMBATANTS: COMPLEMENT}

The complement system is evolutionarily one of the oldest protein cascades of the immune system ( $\sim 500$ million years) [14], and one of the first to be discovered (1890s) [15], yet we still have much to understand to control the complement system for the treatment of diseases. The complement system refers to a set of $\sim 40$ proteins in the blood and surface of cells which recognize foreign substances and dead cells and help clear them [16]. The core function of complement is to distinguish "self" from "non-self," which involves "opsonizing" (binding) complement proteins onto non-self surfaces. Complement opsonization onto foreign surfaces marks the surface for clearance by leukocytes, while releasing into solution protein fragments that orchestrate inflammation and assemble into protein complexes that kill microbes. Thus, complement serves a major role in fighting off the major non-self surfaces that animals have battled for their full billion years: microbes.

Of course, microbes share numerous features with nanocarriers, and thus it should not have come as a surprise that complement's 0.5 billion-year battle would start a new front against nanocarriers. The most obvious similarity between microbes and nanocarriers is size: most pathogenic viruses are $100 \mathrm{~nm}$ (e.g., HIV is $120 \mathrm{~nm}$ ) and most pathogenic bacteria are $\sim 1000 \mathrm{~nm}$ (e.g., E. coli is 1-2,000 nm long). The second key similarity is the possession of surface nucleophiles like primary amines that undergo electrophilic attack by the complement protein C3. Thus, these two shared properties, size and surface nucleophiles, almost guaranteed that complement would open a second front in its battle against nanoparticles in the blood, this time against engineered nanoparticles.

\section{KNOW THE ENEMY COMBATANTS: COMPLEMENT OPSONIZATION AND TOXIN FORMATION}

The complement system may be composed of $\sim 40$ proteins, each with numerous interactions, but the heart of complement is one protein, C3 (Fig. 2). C3 is evolutionarily the oldest of the complement molecules, being found in all deuterostomes (one of the two main branches of Animalia, not including insects, worms, and related) [14], while other complement proteins, such as $\mathrm{C} 4$, are not found in some branches. $\mathrm{C} 3$ is also one of the most abundant proteins in plasma, at $1.2 \mathrm{mg} / \mathrm{mL}$. C3's main role is as an opsonin, covalently bonding to surface nucleophiles (most importantly, primary amines and hydroxyls) via electrophilic attack with its high energy, short-lived thioester bond [16]. This reaction leads to $\mathrm{C} 3$ being broken into 2 pieces: $\mathrm{C} 3 \mathrm{~b}$, which remains covalently bound to the surface, and the peptide $\mathrm{C} 3 \mathrm{a}$, which is free into solution. Both $\mathrm{C} 3 \mathrm{~b}$ and $\mathrm{C} 3 \mathrm{a}$ play major roles in directing the immune system, and in diminishing the benefits of nanomedicine. 


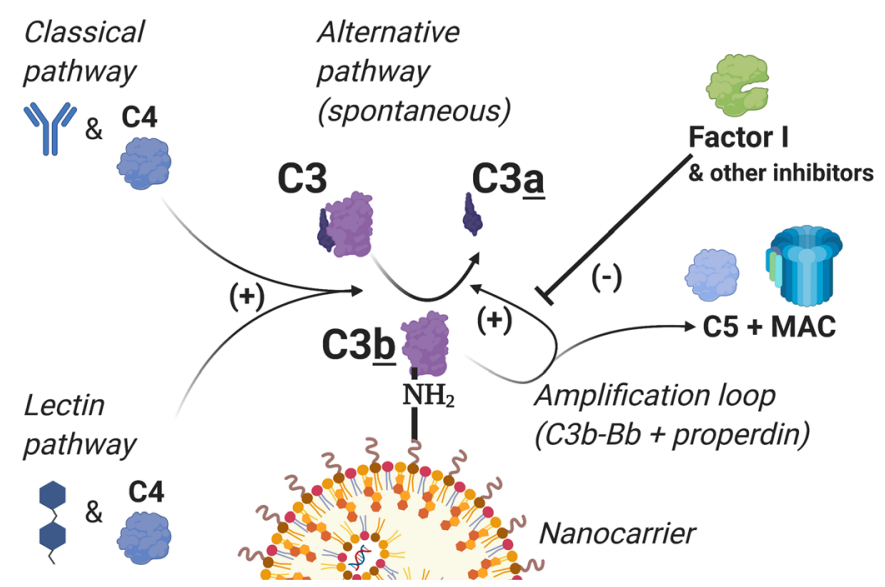

Fig. 2. Map of the enemy: simplified complement pathway as it relates to nanomedicine. A nanoparticle (orange and red semi-circle) is depicted at the bottom, with a free surface nucleophile (depicted here as a primary amine). The heart of the complement pathway is C3's opsonization of a nanoparticle, in which a covalent bond is formed with a surface nucleophile, forming two species: a C3b-nanoparticle adduct, and $\mathrm{C} 3 \mathrm{a}$, which diffuses away into the bulk solution. C3 opsonization can be activated by one of 3 pathways: the classical pathway, in which immunoglobulins, especially when clustered on a surface, activate a C3 convertase containing a fragment of $\mathrm{C} 4$; the lectin pathway, which is similar to the classical pathway but is activated by binding of pattern recognition proteins to foreign polysaccharides; and the alternative pathway, in which $\mathrm{C} 3$ can undergo spontaneous, slow adduct formation, or via the alternative pathway's amplification loop, in which one C3b (as the $\mathrm{C} 3 \mathrm{bBb}+$ properdin complex) catalyzes the addition of a second $\mathrm{C} 3 \mathrm{~b}$ onto a nearby surface nucleophile. These processes are inhibited on animal cells by a series of complement inhibitory proteins, such as Factor I, which are also co-opted by bacteria for the same purpose. Besides the formation of $\mathrm{C} 3 \mathrm{~b}$ and $\mathrm{C} 3 \mathrm{a}$, the other major output of the $\mathrm{C} 3$ opsonization reaction is that $\mathrm{C} 3 \mathrm{bBb}$ (as a dimer) catalyzes the reaction of $\mathrm{C} 5$ to $\mathrm{C} 5 \mathrm{a}$ (an anaphylatoxin) and $\mathrm{C} 5 \mathrm{~b}$, a component of the cell lysing membrane attack complex (MAC)

C3 opsonization is classically said to be initiated by 3 pathways. The classical pathway is activated by clustering of immunoglobulins, especially IgG, onto a surface, as happens with microbes recognized by antibodies. Such clustering leads to a protein complex, $\mathrm{C} 4 \mathrm{~b} 2 \mathrm{a}$, that acts as a $C 3$ convertase, catalyzing the reaction of $\mathrm{C} 3$ to $\mathrm{C} 3 \mathrm{~b}$-surface adducts. The lectin pathway functions similarly, but is initiated by proteins that recognize microbial polysaccharides. Finally, the most important pathway for $\mathrm{C} 3$ activation is the alternative pathway, which contributes up to $80 \%$ of overall C3 activation, even when initiated by classical or lectin pathways $[17,18]$. The alternative pathway is activated by two main routes. First, in tick-over, which occurs at a very slow rate, as $\mathrm{C} 3$ is hydrolyzed to $\mathrm{C} 3\left(\mathrm{H}_{2} \mathrm{O}\right)$. $\mathrm{C} 3\left(\mathrm{H}_{2} \mathrm{O}\right)$ can directly bond to a surface nucleophile, or in solution it can bind Factor $\mathrm{B}$, leading (via Factor D) to the complex $\mathrm{C} 3\left(\mathrm{H}_{2} \mathrm{O}\right) \mathrm{Bb}$, which is a C3 convertase, acting as a catalyst to promote other C3 molecules bonding to surface nucleophiles. Second, in the amplification loop, C3b binds Factor B to become $\mathrm{C} 3 \mathrm{bBb}$, which acts as a very efficient catalyst to bind other C3 molecules to nearby surface nucleophiles. Importantly, the classical, lectin, and tick-over routes all feed into the amplification loop, leading to a (theoretical, never directly observed) spreading of C3b-adduct formation across the surface of microbes. Notably, there are other routes to C3 opsonization, sometimes referred to as the "extrinsic pathway," which includes activation of $\mathrm{C} 3$ to a state similar to $\mathrm{C} 3\left(\mathrm{H}_{2} \mathrm{O}\right)$ when $\mathrm{C} 3$ is physisorbed to a surface (e.g., blood-air interface), or when $\mathrm{C} 3$ is cleaved to $\mathrm{C} 3 \mathrm{~b}$ and $\mathrm{C} 3 \mathrm{a}$ via less specific kinases such as thrombin, which are at high enough concentrations locally sometimes to play a significant role despite their high $\mathrm{K}_{\mathrm{D}}$.

Regardless of the path of activation, $\mathrm{C} 3$ is cleaved into $\mathrm{C} 3 \mathrm{~b}$ and $\mathrm{C} 3 \mathrm{a}$. C3b-surface adducts are rapidly (minutes) converted into iC3b-surface adducts, which promote clearance of microbes and nanocarriers by the RES in two main ways: phagocytosis, as iC3b binds to receptors (CR3, CR4, CRIg) on phagocytes which then phagocytose (eat) the particles; and immune adherence, in which $\mathrm{C} 3 \mathrm{~b}$ binds CR1 on red blood cell surfaces, which transport the captured particles to phagocytes in the spleen and liver. C3b-surface adducts also catalyze the cleave of C5 to two toxic products: C5a, which is a potent anaphylatoxin (promoting anaphylaxislike reactions), and the membrane attack complex (MAC), a multi-protein pore-forming complex that punches holes in cells. While C3b does all this, C3a diffuses into bulk solution, and acts as an anaphylatoxin as well. Thus, the C3 opsonization of a nanocarrier produces two major problems: opsonization marks the nanocarrier for RES uptake, and numerous toxins are released (anaphylatoxins C5a and C3a, 
and the cell-killing MAC). A third problem seems likely, but is not yet proven: opsonization by $\mathrm{C} 3 \mathrm{~b}$-adducts likely foul the targeting moieties on nanocarriers, by steric hindrance.

\section{COLLATERAL CASUALTIES OF THE NANO-WAR: SIDE EFFECTS OF COMPLEMENT-NANOPARTICLE INTERACTIONS}

Above, we identified 3 major problems that complement causes for nanomedicine: opsonization promotes RES uptake; opsonization fouls targeting moieties; and numerous toxins are released (Fig. 3). The first two of these problems should lead to poor biodistribution (a low fraction of nanocarriers deposit in the target organ) and poor pharmacokinetics (the plasma half-life of nanocarriers is shorter than optimal). The biodistribution problem was well illustrated by a meta-analysis of high-quality nanomedicine studies, which showed only $0.7 \%$ (median) of the administered nanocarrier dose is delivered to a solid tumor [19]. What fraction of these poor biodistribution and plasma half-life results is due to complement? The best way to answer this is by studying the biodistribution of nanocarriers in $\mathrm{C} 3$ knockout mice. Unfortunately, this has barely been studied. For PLGA-PEG nanocarriers, there is no difference in serum half-life (biodistribution not reported) in naive vs C3-knockout mice [20]. However, PLGA-PEG nanocarriers often have no surface nucleophiles, except some variants have terminal hydroxyls, which are probably shielded from $\mathrm{C} 3$ by their local hydration shell. Dextran-coated superparamagnetic iron oxide (SPIO) nanoparticles, which barely bound C3 despite binding lectin pathway activator MBL, do not have their halflife changed by C3 knockout [21]. However, for a number of clinical nanocarriers such as LipoDox and Onivyde, it is clear that $\mathrm{C} 3 \mathrm{~b}$ opsonizes the nanocarriers, mostly via pre-formed antibodies binding and activating the classical pathway, though this study did not investigate plasma-half-life [22]. Additionally, we were unable to find any studies investigating how C3b-nanocarrier adduct formation affects targeted nanocarrier avidity to their target. Thus, even in preclinical models, there is a paucity of information on how C3b-adducts affect biodistribution and pharmacokinetics of targeted nanocarriers, nor whether $\mathrm{C} 3 \mathrm{~b}$-adducts foul targeting moieties. However, based on in vitro results, it is absolutely clear that $\mathrm{C} 3 \mathrm{~b}$ heavily opsonizes targeted nanoparticles, so these missing studies must be performed in order for nanoengineers to understand their enemy complement.

There is, however, one problem caused by a complement that is very clearly demonstrated in nanomedicine: the effects of complement-derived toxins. Indeed, there is a clinical syndrome, known as complement-activation-related pseudoallergy (CARPA), which has been thoroughly documented in preclinical models, in clinical trials, and post-

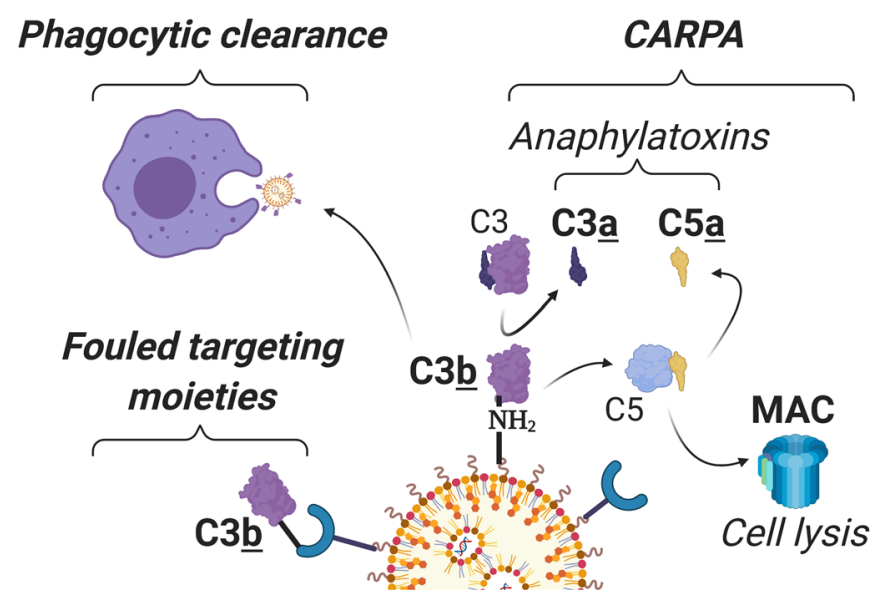

Fig. 3. Casualties of the nano-war: three major deleterious consequences of C3 opsonization. C3 opsonization leads to two products: C3b-nanoparticle adducts, and soluble C3a. C3b has two negative consequences for nanomedicine. First, C3b opsonized nanoparticles are rapidly phagocytosed by phagocytes, with the most important being the liver's Kupffer cells, which can dramatically decrease nanoparticle circulation time. Second, C3b can form adducts on nanocarrier's targeting moieties, and the resulting steric hindrance can decrease the nanocarrier's avidity for its target cells. C3a is an anaphylatoxin, meaning it produces consequences similar to allergic (IgE-mediated) anaphylaxis, such as activation and degranulation of mast cells, and chemotaxis of numerous leukocytes. C5 is broken down into C5a, an anaphylatoxin massively more potent than $\mathrm{C} 3 \mathrm{a}$, and C5b. C5b forms a key component of the membrane attack complex (MAC), which can lyse animal cells by forming a pore in the cell membrane. The combination of the anaphylatoxins and MAC result in the syndrome of CARPA, characterized by transient ( $<30$ minutes) hypotension, urticaria (hives), and bronchospasm. It is also possible that another contribution to CARPA may come from the leukocytes that phagocytose $\mathrm{C} 3 \mathrm{~b}$-opsonized nanoparticles, but this is not yet proven and thus not depicted above 
marketing of nanocarriers [23]. In CARPA, the anaphylatoxins $\mathrm{C} 3 \mathrm{a}$ and $\mathrm{C} 5 \mathrm{a}$ induce an anaphylaxis-like reaction, which in preclinical models results in transient (1030 minutes) hypotension, thrombocytopenia, leukopenia, and bronchospasm. Clinically, symptoms and signs include transient hypotension, wheezing, and urticaria (hives). Nanocarriers implicated in CARPA include the first two FDA-approved nanocarriers, AmBisome and Doxil, as well as several others [23]. CARPA has also been documented in patients infused with several drugs that are not classically thought of as nanomedicine, but are solubilized in a manner that was later shown to involve micelle formation, such as cyclosporine and taxol [23]. The frequency of CARPA is unclear, but for liposomal drugs ranges from $3 \%$ to $45 \%$ [24, 25]. While CARPA is clearly common in nanomedicine, it has not been taken very seriously by many nanoengineers, as it is transient (10-30 minutes), rarely if ever results in death, occurs usually in outpatient cancer patients who can tolerate brief hypotension, and can be partially treated with antihistamines and IV fluid bolus. However, if nanomedicine is going to be used more widely, nanoengineers must (1) study targeted nanocarriers, which are more likely than bare nanocarriers to induce severe CARPA as their targeting moieties are better substrates for C3; and (2) study CARPA in disease states which are more likely susceptible to the consequences of CARPA, such as patients in the ICU with septic shock or stroke, which are patient populations known to poorly tolerate even transient hypotension. Such studies will better define where the enemy, complement, is most likely to win battles.

\section{VULNERABILITIES TO ENEMY ATTACK: WHAT NANOPARTICLE SURFACE FEATURES ARE MOST SUSCEPTIBLE TO COMPLEMENT DEPOSITION?}

Nanocarriers are made out of a wide range of materials, and it is not possible to survey every material and its C3b opsonization. Instead, we may address the following points: (1) What general nanomaterial properties lead to C3 opsonization? (2) How do other opsonins interact with complement and affect $\mathrm{C} 3 \mathrm{~b}$ opsonization?

Size is the most pervasively studied property of nanocarriers, and this has been analyzed for $\mathrm{C} 3 \mathrm{~b}$ opsonization. For the most clinically translated nanocarrier, liposomes, it is clear that very large liposomes (several micron, multilamellar vesicles) activate complement much more than the clinically used $\sim 100 \mathrm{~nm}$ liposomes, which on their own did not activate complement [26]. Larger size also improves $\mathrm{C} 3 \mathrm{~b}$ opsonization of protein-coated nanoparticles, at least comparing ovalbumin-conjugated $5 \mathrm{~nm}$ vs $50 \mathrm{~nm}$ gold nanoparticles [27]. Similarly, on silica nanoparticles, whose surface hydroxyls make C3 one of the particle's top 3 opsonins, larger size also increased $\mathrm{C} 3$ opsonization at a very small size, comparing 10 vs $20 \mathrm{~nm}$ particles, but actually decreased for $70 \mathrm{~nm}$ particles [28]. Thus, the overall trend is clear that the larger size of nanoparticles augments complement deposition, though the mechanism is not yet determined.

Nanoparticles have several other quantifiable properties, but only a couple have been studied for their complement opsonization ability. Probably the second most tested property of nanocarriers is zeta potential, which is reasonably understood as surface charge. Among liposomes of the typical $\sim 100 \mathrm{~nm}$ diameter, both positive and negative zeta potentials potentiate complement activation, while neutral liposomes are barely able to activate complement [8]. Interestingly, negatively charged liposomes activate the classical pathway, while positively charged nanoparticles activate the alternative pathway [8]. Finally, the last property that has been studied in depth is membrane fluidity, where it was found that unsaturated lipids and cholesterol in liposomes, both of which are known to increase membrane fluidity, increase complement opsonization [8].

Complement is not the only combatant battling nanomedicine, as there are numerous other opsonins. Importantly, these other opsonins strongly determine complement opsonization. First, it was shown that on SPIONs conjugated to dextran strands, non-complement opsonins (e.g., fibrinogen, albumin, and immunoglobulin) intercalated in between the dextran strands creating a non-complement corona, and C3 then opsonized the proteins of that non-complement corona [29]. Second, it was shown that in human plasma, the opsonin in the non-complement corona that is responsible for nearly all the $\mathrm{C} 3 \mathrm{~b}$ deposition is $\mathrm{IgG}$ [22]. This was true not just of SPIONs, but also of FDA-approved liposomes (Onivyde and LipoDox), and it was true regardless of which complement pathway (classical, lectin, alternative) was necessary for full complement activation. Thus, nanoparticles do not only have to fight against complement, but also other opsonins which are collaborators with complement.

\section{FIGHTING BACK: WHAT NANOCARRIER STRATEGIES HAVE BEEN TAKEN TO AVOID COMPLEMENT?}

Many polymer coatings have been applied to nanoparticles to improve plasma circulation time, and it was later revealed that many of these coatings also decrease C3 opsonization. This was well-studied in SPIONs, which are nanoparticles used as MRI contrast agents. Several of these were tested for complement activation, and found carboxymethyldextran and dextran-coated SPIONs caused significant complement activation, while citric acid, phosphatidylcholine, starch, and chitosan-coated SPIONs had no such effect [30]. However, the best-studied polymer coating is PEG, which has been extensively shown to reduce C3 opsonization. PEG density matters, and increasing the PEG density nearly linearly decreased $\mathrm{C} 3 \mathrm{~b}$ surface adducts, as measured by LC/MS [31]. The same was true of PEG on PLGA nanoparticles [32]. However, PEG does not completely eliminate C3 adducts. Further, when targeting moieties are conjugated onto the surface of nanocarriers, the PEG almost certainly does not effectively prevent $\mathrm{C} 3 \mathrm{~b}$ opsonization, though this has not been studied effectively. Finally, as $\sim 25 \%$ of humans have anti-PEG antibodies [33], which almost certainly will lead to $\mathrm{C} 3 \mathrm{~b}$ opsonization. Thus, PEGylation has helped in the battle against complement, but it has certainly not won the war.

The other approach to combating C3 opsonization of nanocarriers has been to infuse complement inhibitors before or along with nanocarriers. For example, SPIONs conjugated to targeting antibodies elicit strong $\mathrm{C} 3 \mathrm{~b}$ opsonization in whole 
blood and are therefore taken up rapidly by leukocytes, but multiple C3-convertase inhibitors (compstatin, soluble C35, and various fragments of CD59) blocked $\mathrm{C} 3 \mathrm{a}$ and $\mathrm{C} 5 \mathrm{a}$ production, and prevented $99 \%$ of leukocyte uptake of the SPIONs [34, 35]. Additionally, infusion of the natural, soluble complement inhibitor Factor $\mathrm{H}$, dramatically reduced complement activation in whole blood exposed to the clinically used nanocarriers AmBisome (a liposome) and Cremophor EL (a polymeric detergent used to solubilize many chemotherapeutics, which forms micelles) [36]. Thus far, these products have only been tested in vitro, and so require further in vivo testing, and perhaps engineering specific to nanomedicine.

\section{WEARING THE ENEMY'S UNIFORM: UTILIZING COMPLEMENT TO AID NANOMEDICINE}

While most nanoengineering will continue to focus on avoiding the deleterious aspects of complement, we recently found that complement opsonization can also be utilized to create a therapy. We screened a large array of nanomaterials for uptake into the lungs of mice that had received nebulized lipopolysaccharide (LPS), a model of acute respiratory distress syndrome (ARDS), the lung inflammation that kills in COVID-19 [37]. We found that nanoparticles with agglutinated surface protein (NAPs; e.g., albumin nanoparticles), but not nanoparticles with near-crystalline arrangement of surface proteins (e.g., ferritin nanocages), had a strong tropism for marginated neutrophils in the lungs of the LPS mice. We then found that NAP uptake in marginated neutrophils required $\mathrm{C} 3$ opsonization, and that nearcrystalline protein nanoparticles lack such C3 opsonization. Most intriguing, we found that NAP-liposomes (liposomes with clustered surface proteins) not only had tropism for the marginated neutrophils, but also caused the neutrophils to demarginate (leave their residence in the lung capillaries) and travel to the spleen. This led to a dramatic therapeutic effect of the ARDS-like phenotypes. Thus, complement opsonized nanoparticles acted like decoys, which distracted marginated neutrophils from their pro-inflammatory role, and thereby ameliorated a major inflammatory disease.

\section{CONCLUSIONS AND FUTURE DIRECTIONS OF THE NANO-WAR}

After $>30$ years of studying, the war between nanomedicine and complement much has been learned. It is clear that complement deposition on nanocarriers produces the toxins underlying the CARPA reaction, which while minor in most patient populations, might prove a major barrier to using nanomedicine in severely ill patients in the ICU. Additionally, complement opsonization is extensive on many nanocarriers, especially those with targeting moieties, and this leads to decreased plasma-half-life of the nanocarrier and likely fouling of the targeting moieties' avidity (though the latter is unproven). Further, we now know that complement conspires with other opsonins, especially $\operatorname{IgG}$, that physisorb onto nanoparticles and, once deposited, form the nidus of complement activation. Finally, we have learned some material properties that can help decrease complement opsonization, including smaller size, neutral charge, less surface fluidity, coating by select polymers such as PEG. Unfortunately, these properties decrease, but do not eliminate complement deposition, and heavily constrain nanocarrier design, especially reducing targeting moiety selection.

The future of the nano-war is clearly to improve our understanding of complement and start building more specific weapons. We must conduct several studies of the enemy complement, including studying CARPA in vulnerable patient populations, such as ICU patients, to ensure we do cause harm to them with early introduction of nanomedicine; test complement interaction not simply with bare nanocarriers, but also with targeted nanocarriers, who's targeting moieties (e.g., antibodies and their derivatives) make them particularly vulnerable to complement; and study why certain nanoparticle properties, such as increased size and surface fluidity, augment complement activation. After such studies, we can design improved nanocarriers. Perhaps, we can take inspiration from the complement evasion strategies of bacteria, which bind human complement inhibitory proteins (e.g., $\mathrm{C} 4 \mathrm{BP}, \mathrm{FH}$, and vitronectin) [38]. Combined with improved stealth polymer, such as several zwitterionic polymers [39], these techniques might inhibit complement opsonization enough to prevent the deleterious effects above.

Beyond our goal of simply keeping the enemy at bay, two other goals are emerging in the nano-war on complement. First, it appears useful to employ complement as a cloak, in order to gain entry into the leukocytes responsible for the negative effects of acute inflammation. This was shown by the complement-opsonized liposomes that had strong tropism for pulmonary marginated neutrophils, and once inside the cells, caused them to leave the lungs and thereby strongly ameliorated disease models of ARDS [37]. Second, nanomedicine is actually well-poised for making inroads as complement inhibitors. Numerous complement inhibitors are in clinical trials, and for all of them, immunosuppression is a side effect. Nanomedicine's ability to control pharmacokinetics of drugs could perhaps solve this problem, by allowing a quick burst of complement suppression followed by rapid clearance, for treating diseases like stroke, where complement is dangerous in the first several hours after reperfusion, but patients are at high risk of infection for the next few weeks. Thus, the nano-war has evolved from an all-out attempt to suppress complement, to a battle to control complement.

\section{ACKNOWLEDGMENTS}

We would like to thank Jacob W. Myerson, Vladimir R Muzykantov, and Jia Nong for their numerous discussions on this topic.

\section{AUTHORS' CONTRIBUTIONS}

Both authors contributed to the literature search, synthesize of ideas, and writing.

\section{FUNDING}

This work was supported by NIH K08-HL-138269-01 and R01-HL-153510-01. 


\section{DECLARATIONS}

Conflict of Interest J.S.B. is listed on patents pertaining to complement's role in nanomedicine, which are owned by the University of Pennsylvania.

\section{REFERENCES}

1. Williams KJ. The introduction of "chemotherapy" using arsphenamine - the first magic bullet. J R Soc Med. 2009;102:343-8.

2. Bangham AD, Horne RW. Negative staining of phospholipids and their structural modification by surface-active agents as observed in the electron microscope. J Mol Biol. 1964;8:660-8.

3. Torchilin VP, Khaw BA, Smirnov VN, Haber E. Preservation of antimyosin antibody activity after covalent coupling to liposomes. Biochem Biophys Res Commun. 1979;89:1114-9.

4. Laginha KM, Verwoert S, Charrois GJR, Allen TM. Determination of doxorubicin levels in whole tumor and tumor nuclei in murine breast cancer tumors. Clin Cancer Res. 2005;11:6944-9.

5. Zhang X, Goel V, Attarwala H, Sweetser MT, Clausen VA, GJ Robbie. Patisiran Pharmacokinetics, Pharmacodynamics, and Exposure-Response Analyses in the Phase 3 APOLLO Trial in Patients With Hereditary Transthyretin-Mediated (hATTR) Amyloidosis. J Clin Pharmacol. 2020;60(1):37-49. Available from: https://doi.org/10.1002/jcph.1480

6. Gustafson HH, Holt-Casper D, Grainger DW, Ghandehari H. Nanoparticle uptake: the phagocyte problem. Nano Today. 2015;10:487-510.

7. Artursson P, Mårtensson IL, Sjöholm I. Biodegradable microspheres. III: some immunological properties of polyacryl starch microparticles. J Pharm Sci. 1986;75:697-701.

8. Chonn A, Cullis PR, Devine DV. The role of surface charge in the activation of the classical and alternative pathways of complement by liposomes. J Immunol. 1991;146:4234-41.

9. Scieszka JF, Maggiora LL, Wright SD, Cho MJ. Role of complements $\mathrm{C} 3$ and $\mathrm{C} 5$ in the phagocytosis of liposomes by human neutrophils. Pharm Res. 1991;8:65-9.

10. Alving CR, Wassef NM. Complement-dependent phagocytosis of liposomes: suppression by "stealth" lipids. J Liposome Res. Taylor \& Francis. 1992;2:383-95.

11. Borchard G, Kreuter J. Interaction of serum components with poly(methylmethacrylate) nanoparticles and the resulting body distribution after intravenous injection in rats. J Drug Target. 1993;1:15-9.

12. Devine DV, Wong K, Serrano K, Chonn A, Cullis PR. Liposome-complement interactions in rat serum: implications for liposome survival studies. Biochim Biophys Acta. 1994;1191:43-51.

13. Allémann E, Gravel P, Leroux JC, Balant L, Gurny R. Kinetics of blood component adsorption on poly(D,L-lactic acid) nanoparticles: evidence of complement C3 component involvement. J Biomed Mater Res. 1997;37:229-34.

14. Nonaka M. Evolution of the complement system. Curr Opin Immunol. 2001;13:69-73.

15. Walport MJ. Complement. First of two parts. N Engl J Med. 2001;344:1058-66.

16. Ricklin D, Reis ES, Mastellos DC, Gros P, Lambris JD. Complement component C3 - The "Swiss Army Knife" of innate immunity and host defense. Immunol Rev. 2016;274:3358 .

17. Harboe M, Ulvund G, Vien L, Fung M, Mollnes TE. The quantitative role of alternative pathway amplification in classical pathway induced terminal complement activation. Clin Exp Immunol. Wiley. 2004;138:439-46.

18. Harboe M, Garred P, Karlstrøm E, Lindstad JK, Stahl GL, Mollnes TE. The down-stream effects of mannan-induced lectin complement pathway activation depend quantitatively on alternative pathway amplification. Mol Immunol. 2009;47:373-80.

19. Wilhelm S, Tavares AJ, Dai Q, Ohta S, Audet J, Dvorak HF, et al. Analysis of nanoparticle delivery to tumours. Nat Rev Mater. Macmillan Publishers Limited. 2016;1:16014.

20. Bertrand N, Grenier P, Mahmoudi M, Lima EM, Appel EA, Dormont $\mathrm{F}$, et al. Mechanistic understanding of in vivo protein corona formation on polymeric nanoparticles and impact on pharmacokinetics. Nat Commun. 2017;8:777.

21. Simberg D, Park J-H, Karmali PP, Zhang W-M, Merkulov S, McCrae K, et al. Differential proteomics analysis of the surface heterogeneity of dextran iron oxide nanoparticles and the implications for their in vivo clearance. Biomaterials. 2009;30:3926-33.

22. Vu VP, Gifford GB, Chen F, Benasutti H, Wang G, Groman EV, et al. Immunoglobulin deposition on biomolecule corona determines complement opsonization efficiency of preclinical and clinical nanoparticles. Nat Nanotechnol. 2019;14:260-8.

23. Szebeni J. Complement activation-related pseudoallergy: a stress reaction in blood triggered by nanomedicines and biologicals. Mol Immunol. 2014;61:163-73.

24. Szebeni J. Complement activation-related pseudoallergy caused by liposomes, micellar carriers of intravenous drugs, and radiocontrast agents. Crit Rev Ther Drug Carrier Syst. Begell House. 2001;18:567-606.

25. Szebeni J, Fontana JL, Wassef NM, Mongan PD, Morse DS, Stahl GL, et al. Liposome-induced and complement-mediated cardiopulmonary distress in pigs as a model of pseudo-allergic reactions to liposomal drugs. Mol Immunol. Elsevier BV. 1998;35:401.

26. Szebeni J, Baranyi L, Savay S, Bodo M, Morse DS, Basta M, et al. Liposome-induced pulmonary hypertension: properties and mechanism of a complement-mediated pseudoallergic reaction. Am J Physiol Heart Circ Physiol. 2000;279:H1319-28.

27. Zhang Y-N, Lazarovits J, Poon W, Ouyang B, Nguyen LNM, Kingston BR, et al. Nanoparticle size influences antigen retention and presentation in lymph node follicles for humoral immunity. Nano Lett. 2019;19:7226-35.

28. Tenzer S, Docter D, Rosfa S, Wlodarski A, Kuharev J, Rekik A, et al. Nanoparticle size is a critical physicochemical determinant of the human blood plasma corona: a comprehensive quantitative proteomic analysis. ACS Nano. 2011;5:7155-67.

29. Chen F, Wang G, Griffin JI, Brenneman B, Banda NK, Holers $\mathrm{VM}$, et al. Complement proteins bind to nanoparticle protein corona and undergo dynamic exchange in vivo. Nat Nanotechnol. Nature Publishing Group. 2017;12:387-93.

30. Fülöp T, Nemes R, Mészáros T, Urbanics R, Kok RJ, Jackman JA, et al. Complement activation in vitro and reactogenicity of low-molecular weight dextran-coated SPIONs in the pig CARPA model: correlation with physicochemical features and clinical information. J Control Release. 2018;270:268-74.

31. Walkey CD, Olsen JB, Guo H, Emili A, Chan WCW. Nanoparticle size and surface chemistry determine serum protein adsorption and macrophage uptake. J Am Chem Soc. 2012;134:2139-47.

32. Partikel K, Korte R, Stein NC, Mulac D, Herrmann FC, Humpf $\mathrm{H}-\mathrm{U}$, et al. Effect of nanoparticle size and PEGylation on the protein corona of PLGA nanoparticles. Eur J Pharm Biopharm. 2019;141:70-80.

33. Garay RP, El-Gewely R, Armstrong JK, Garratty G, Richette P. Antibodies against polyethylene glycol in healthy subjects and in patients treated with PEG-conjugated agents. Expert Opin Drug Deliv. Informa Healthcare. 2012;9:1319-23.

34. Gaikwad H, Li Y, Gifford G, Groman E, Banda NK, Saba L, et al. Complement inhibitors block complement C3 opsonization and improve targeting selectivity of nanoparticles in blood. Bioconjug Chem. 2020;31:1844-56.

35. Gifford G, Vu VP, Banda NK, Holers VM, Wang G, Groman $\mathrm{EV}$, et al. Complement therapeutics meets nanomedicine: overcoming human complement activation and leukocyte uptake of nanomedicines with soluble domains of CD55. J Control Release. 2019;302:181-9.

36. Mészáros T, Csincsi ÁI, Uzonyi B, Hebecker M, Fülöp TG, Erdei A, et al. Factor $\mathrm{H}$ inhibits complement activation induced 
by liposomal and micellar drugs and the therapeutic antibody rituximab in vitro. Nanomedicine. 2016;12:1023-31.

37. Myerson JW, Patel PN, Habibi N, Walsh LR, Lee Y-W, Luther DC, et al. Supramolecular Organization Predicts Protein Nanoparticle Delivery to Neutrophils for Acute Lung Inflammation Diagnosis and Treatment [Internet]. bioRxiv. 2020 [cited 2020 Apr 20]. p. 2020.04.15.037564. Available from: https:// www.biorxiv.org/content/10.1101/2020.04.15.037564v1

38. Blom AM, Hallström T, Riesbeck K. Complement evasion strategies of pathogens-acquisition of inhibitors and beyond. Mol Immunol. Elsevier BV. 2009;46:2808-17.
39. Fam SY, Chee CF, Yong CY, Ho KL, Mariatulqabtiah AR, Tan WS. Stealth Coating of Nanoparticles in Drug-Delivery Systems. Nanomaterials (Basel). 2020;10(4):787. Available from: https://doi.org/10.3390/nano10040787

Publisher's Note Springer Nature remains neutral with regard to jurisdictional claims in published maps and institutional affiliations. 